\title{
Effect of local electron-electron correlation in hydrogen-like impurities in Ge
}

\author{
H. Sims, ${ }^{1}$ E. R. Ylvisaker, ${ }^{2}$ E. Şaşıŏglu,${ }^{3}$ C. Friedrich,${ }^{3}$ S. Blügel, ${ }^{3}$ and W. E. Pickett ${ }^{2}$ \\ ${ }^{1}$ Center for Materials for Information Technology (MINT) and Department of Physics, University of Alabama, Tuscaloosa, \\ Alabama 35487, USA \\ ${ }^{2}$ Department of Physics, University of California, Davis, Davis, California 95616, USA \\ ${ }^{3}$ Peter Grünberg Institut and Institute for Advanced Simulation, Forschungszentrum Jülich and JARA, 52425 Jülich, Germany
}

(Received 18 February 2013; published 15 May 2013)

\begin{abstract}
We have studied the electronic and local magnetic structure of the hydrogen interstitial impurity at the tetrahedral site in diamond-structure Ge, using an empirical tight binding + dynamical mean-field-theory approach because within the local-density approximation (LDA) Ge has no gap. We first establish that within LDA the $1 s$ spectral density bifurcates due to entanglement with the four neighboring $s p^{3}$ antibonding orbitals, providing an unanticipated richness of behavior in determining under what conditions a local moment hyperdeep donor or Anderson impurity will result, or on the other hand, a gap state might appear. Using a supercell approach, we show that the spectrum, the occupation, and the local moment of the impurity state displays a strong dependence on the strength of the local on-site Coulomb interaction $U$, the H-Ge hopping amplitude, the depth of the bare $1 s$ energy level $\epsilon_{H}$, and we address to some extent the impurity concentration dependence. In the isolated impurity, strong interaction regime a local moment emerges over most of the parameter ranges, indicating magnetic activity, and spectral density structure very near (or in) the gap suggests possible electrical activity in this regime.
\end{abstract}

DOI: 10.1103/PhysRevB.87.195120

PACS number(s): 71.10.-w, 71.20.Nr, 71.55.Cn

\section{INTRODUCTION}

Due to their importance in electronics technology, isolated defects in semiconductors and insulators have a long history. Low doping levels, arising from isolated shallow defects, provide the carriers that make semiconductors a dominant technology in today's pervasive electronics environment. The primary shallow defects in the more important semiconductors for most applications ( $\mathrm{Si}, \mathrm{GaAs}, \mathrm{Ge}$ ) have been extensively studied and research turned to the study of deep levels (states with energies well away from the edges, deep within the gap). An exploration by Haldane and Anderson ${ }^{1}$ demonstrated, considering intra-atomic repulsion using the multiorbital Anderson impurity in a model semiconductor treated in mean field, how multiple charge states can arise and be confined within the semiconducting gap. These charge states will, except accidentally, be deep levels, and when providing a carrier to the conduction band through thermal or electromagnetic excitation, they become deep donor levels.

One of the suspected deep donor impurities in semiconductors, and seemingly the simplest, is interstitial $\mathrm{H}$ in an elemental semiconductor. $\mathrm{Ge}$ and $\mathrm{Si}$ can be prepared ultrapure, and $\mathrm{H}$ possibly is the most common remaining impurity. In work that will be discussed in more detail later, Pickett, Cohen, and $\mathrm{Kittel}^{2}$ (PCK) provided evidence that interstitial $\mathrm{H}$ produces a hyperdeep donor level in $\mathrm{Ge}$, with the $\mathrm{H} 1 s$ donor state lying not within the gap but perhaps located as deep as $6 \mathrm{eV}$ below the gap, near the center of the valence bands. Their hands-on, self-consistent mean-field treatment in the spirit of correlated band theory $(\mathrm{LDA}+\mathrm{U})$ methods leaves much yet to be decided.

PCK provided a synopsis of the earlier models that had been applied to this $\mathrm{H}$ impurity question. Several H-related defects have been observed ${ }^{3-7}$ in $\mathrm{Ge}$, and most seem to be defect complexes in which $\mathrm{H}$ is involved, rather than simply isolated $\mathrm{H}$ impurities. However, local vibrations were observed for isolated $\mathrm{H}$, identified as (near) bond-centered and in the antibonding or tetrahedral sites, ${ }^{8,9}$ which is the impurity of interest here. Similar questions exist for $\mathrm{H}$ impurities in the isovalent semiconductors $\mathrm{Si}^{10}$ and diamond.

Since that early work, a few model studies have addressed the effects of local interactions at a single orbital impurity in a semiconducting host. Yu and Guerrero investigated a one-dimensional Anderson model with an impurity using the density matrix renormalization group approach. ${ }^{11}$ The strength of the hybridization compared to the semiconducting gap determined whether the doped-hole density remained localized near the impurity or instead spread over many sites (25 sites in their study). Additional holes were found to be spread throughout the system, avoiding the impurity region. The $\mathrm{H}$ in Ge problem is a physical realization of the gapped Anderson impurity model (GAIM) studied by Galpin and Logan. ${ }^{12,13}$ They addressed the GAIM with a self-consistent perturbation theory extended to all orders and concluded that for the half-filled case such as we are interested in here-neutral $\mathrm{H}$ in undoped $\mathrm{Ge}$ - for any nonzero gap the interacting system is not perturbatively connected to the noninteracting system. This broad claim calls to mind the classic result of Kohn and Majumdar — separate but related, and with different connotations - that the properties of such a system (in the noninteracting case) are analytic in the strength of a local potential that drives a bound gap state across the gap edge to become a resonant state in the continuum. ${ }^{14}$

From the earliest electronic structure studies involving $\mathrm{H}$ impurities in $\mathrm{Ge}$, most of the focus has been on defect complexes incorporating $\mathrm{H}$ with vacancies and other impurities. Model studies ${ }^{15,16}$ gave way to a number of densityfunctional theory (DFT) based studies; see Refs. 6 and 7 for representative work. DFT studies of isolated $\mathrm{H}$ in Ge and other semiconductors have also been reported, ${ }^{7,17-19}$ giving indications that $\mathrm{H}$ provides in Ge a shallow donor or shallow acceptor, depending on its position (see above), or that it is an example of a negative- $U$ system because of instability of its 
neutral state. These scenarios, formulated within a quantum theory of energetics (DFT) but a one-electron picture of the spectrum, contrast strikingly with the deep donor possibility posed by PCK. Most of the existing studies confine their focus to energetics of the H-in-Ge system and on "energy level" positions, without an exposition of the spectral distribution of the $\mathrm{H} 1 s$ weight.

While the $\mathrm{H}$ impurity in an elemental semiconductor is the most primitive realization of the impurity problem, this type of system has not seen a material-specific treatment of the dynamical correlations that will influence its electronic structure and excitation spectrum. In this paper we provide results of a dynamical mean-field-theory (DMFT) treatment ${ }^{20-22}$ that sheds light on several of the primary issues.

\section{METHOD OF CALCULATION}

\section{A. Supercells: Host electronic structure}

Interstitial $\mathrm{H}$ in intrinsic $\mathrm{Ge}$ presents a seemingly simple system: a single half-filled $1 s$ orbital hybridized with a semiconducting bath. A neutral $\mathrm{H}$ impurity (our interest here) adds one electron that is expected to be accommodated in an additional "state" within the gap or the valence band and most likely the latter, since there has been no signature of an electrically and magnetically active gap state.

Anticipating the disturbance (in density, in screened potential) in an insulator to be localized, we adopt a supercell representation of the impurity. We consider a single interstitial hydrogen atom in the tetrahedrally symmetric antibonding position in $\mathrm{Ge}$, both in a single (periodic) conventional cubic diamond-structure supercell (containing $8 \mathrm{Ge}$ atoms, denoted $\left.\mathrm{HGe}_{8}\right)$ and in a $2 \times 2 \times 2$ supercell of conventional cells (containing $64 \mathrm{Ge}$ atoms and denoted $\mathrm{HGe}_{64}$ ). Choosing two different supercell sizes allows us to further study the influence of effective $\mathrm{H}-\mathrm{Ge}$ hybridization beyond simply reducing the hopping matrix elements and tests to what degree the influence of the $\mathrm{H}$ impurity is spatially localized. There is vibrational evidence $^{8,9}$ that $\mathrm{H}$ sits off the tetrahedral site along a [111] direction, thus closer to one of the four Ge ions than the others, giving it only one Ge nearest neighbor. We do not treat that possibility here, although the methods we use can be applied to that case. Due to a number of uncertainties about materials parameters (and the local-density approximation [LDA] gap problem due to the small gap of Ge), we vary the parameters that are not well established, with the goal of obtaining a more general picture of the behavior of a "H-like" interstitial in an elemental tetrahedral semiconductor.

One challenge is to deal with the gap underestimation in LDA. In Ge, the LDA gap is slightly negative, in contrast to the observed gap of $0.8 \mathrm{eV}$. Since our objective is an initial investigation of dynamical correlations at the $\mathrm{H}$ site, we adopt the simplest representation of the Ge electronic structure. Semiconducting Ge will be modeled here using an empirical nearest-neighbor Slater-Koster (S-K) tight-binding model (eTB) consisting of four Wannier orbitals (one $s$ and three $p$ orbitals) per Ge with parameters obtained from the work of Newman and Dow. ${ }^{23}$ The H-Ge hopping parameters are taken from the work of Pandey. ${ }^{24}$

\section{B. DMFT parameters}

There are, inevitably for the current stage of DMFT theory, two parameters that are not known a priori: the Coulomb interaction $U$ and the bare on-site $1 s$ energy $\epsilon_{H}$ with respect to the Ge band gap. For the single-orbital problem there is no Hund's rule $J_{H}$ interaction to be concerned about, nor multiplet effects. In fact, for the isolated $\mathrm{H}$ interstitial the DMFT result is exact to within numerical uncertainties. While $\mathrm{H}-\mathrm{Ge}$ hybridization amplitudes could be extracted from firstprinciples DFT calculations, since the gap problem in LDA leads us to use an eTB model of the Ge electronic structure, we use eTB hopping amplitudes that were derived in the same spirit.

The hydrogen on-site energy $\epsilon_{H}$ is varied as part of this investigation, guided somewhat by the LDA calculations reported in Sec. III. Within LDA, where there are no parameters, the $1 s$ spectral density for $\mathrm{H}$ in the tetrahedral site unexpectedly bifurcates, so there is no clear point of reference for fixing $\epsilon_{H}$. This splitting is a result of the rather strong hybridization of the $1 s$ orbital with the $s p^{3}$ antibonding orbitals of the four surrounding Ge atoms. LDA includes, for a localized state such as a weakly hybridized $1 s$ orbital, a spurious self-interaction that raises the LDA site energy above what is presumed in a LDA + DMFT calculation, providing an extra challenge for determining $\epsilon_{H}$. The $\mathrm{H} 1 s$ orbital likely is not a really strongly localized state in Ge, but we expect that $\epsilon_{H}=-4 \mathrm{eV}$ should be regarded as an upper bound of the bare $1 s$ level. We use the two values -5 and $-8 \mathrm{eV}$ to span the reasonable range of this parameter. With regard to the on-site energy, we use the bottom of the gap as the zero of energy throughout this paper.

The bare (i.e., unscreened) on-site repulsion $U_{0}$ for an isolated $\mathrm{H} 1 s$ orbital is $\frac{5}{4} \mathrm{Ry}=17.01 \mathrm{eV}$. This is perhaps surprisingly small for what might seem to be a very small orbital: the $1 s$ orbital of the smallest atom. However, it becomes reasonable once it is recognized that the $1 s$ radial density $4 \pi r^{2} \rho(r)$ peaks at $1 a_{0}$, whereas the comparable quantity in $3 d$ cations peaks at $0.6-0.9 a_{0}$ and has $U_{0} \approx$ 25-30 eV. Screening at a large interstitial site in a small gap insulator is hard to estimate, with no comparable values in the literature. We investigate screened values $U=7$ and $12 \mathrm{eV}$ to span the likely range. $U=12 \mathrm{eV}$ is not much smaller than the unscreened, isolated $\mathrm{H}$ value and should allow the examination of the strong interaction regime. The choice of $7 \mathrm{eV}$ has specific interest: PCK argued ${ }^{2}$ that a lone $\mathrm{H} 1 s$ state would have a bare interaction energy on the order of $1 \mathrm{Ry}$ (our analytic value is actually $17 \mathrm{eV}$ ) and that reduction by screening in an insulator would leave a substantial interaction strength of $6-7 \mathrm{eV}$. This amount of reduction, and more, has over the intervening years become commonplace in understanding the effective values of $U$ in transition metal oxides.

\section{Constrained random-phase approximation}

Although we vary both parameters in our impurity Hamiltonian (see Sec. II E), as well as the $\mathrm{H}-\mathrm{Ge}$ hybridization, it is still beneficial to understand the physical value of the interactions in order to both analyze the validity of our range of considered $U$ and of the predictions of PCK and to motivate and guide future material-specific studies. We do this by employing the constrained random-phase approximation (cRPA), ${ }^{25-28}$ 
performed within the full-potential linearized augmentedplane-wave (FLAPW) method using maximally localized Wannier functions (MLWFs). ${ }^{29,30}$ We use the FLAPW method as implemented in the FLEUR code ${ }^{31}$ with the PerdewBurke-Ernzerhof (PBE) exchange-correlation potential ${ }^{32}$ for the ground-state calculations. MLWFs are constructed with the WANNIER90 code. ${ }^{33,34}$ The effective Coulomb potential is calculated within the recently developed cRPA method implemented in the SPEX $\operatorname{code}^{35}$ (for further technical details see Refs. 29, 36, and 37). We use a grid of $6 \times 6 \times 6 k$ points in our $\mathrm{HGe}_{8} \mathrm{cRPA}$ calculations.

The cRPA consists of first writing the polarizability

$$
\begin{aligned}
P\left(\mathbf{r}, \mathbf{r}^{\prime}, \omega\right)= & \sum_{\sigma} \sum_{n}^{\text {occ }} \sum_{m}^{\text {unocc }}\left[\frac{\psi_{\sigma n}^{*}(\mathbf{r}) \psi_{\sigma m}(\mathbf{r}) \psi_{\sigma m}^{*}\left(\mathbf{r}^{\prime}\right) \psi_{\sigma n}\left(\mathbf{r}^{\prime}\right)}{\omega-\varepsilon_{\sigma m}+\varepsilon_{\sigma n}+i \delta}\right. \\
& \left.-\frac{\psi_{\sigma n}(\mathbf{r}) \psi_{\sigma m}^{*}(\mathbf{r}) \psi_{\sigma m}\left(\mathbf{r}^{\prime}\right) \psi_{\sigma n}^{*}\left(\mathbf{r}^{\prime}\right)}{\omega+\varepsilon_{\sigma m}-\varepsilon_{\sigma n}-i \delta}\right]
\end{aligned}
$$

where $\psi_{i}$ and $\varepsilon_{i}$ are the DFT wave functions and their eigenvalues, and $\sigma$ runs over both spin channels. If one separates $P$ into $P_{l}$, containing the correlated orbitals, and $P_{r}$ containing the rest, and if one considers the unscreened Coulomb operator $v$, one can write ${ }^{25-29}$

$$
U=\left[1-v P_{r}\right]^{-1} v .
$$

The matrix elements of the effective Coulomb potential $U$ in the MLWF basis are given by

$$
\begin{aligned}
U_{\mathbf{R} n_{1} n_{3} ; n_{4} n_{2}}(\omega)= & \iint w_{n_{1} \mathbf{R}}^{*}(\mathbf{r}) w_{n_{3} \mathbf{R}}(\mathbf{r}) U\left(\mathbf{r}, \mathbf{r}^{\prime} ; \omega\right) \\
& \times w_{n_{4} \mathbf{R}}^{*}\left(\mathbf{r}^{\prime}\right) w_{n_{2} \mathbf{R}}\left(\mathbf{r}^{\prime}\right) d^{3} r d^{3} r^{\prime},
\end{aligned}
$$

where $w_{n \mathbf{R}}(\mathbf{r})$ is the MLWF at site $\mathbf{R}$ with orbital index $n$ and $U\left(\mathbf{r}, \mathbf{r}^{\prime} ; \omega\right)$ is calculated within the cRPA.

In our calculations, we choose the Ge $4 s 4 p$ and the $\mathrm{H}$ $1 s$ orbitals as our correlated subspace. This is motivated by several considerations. First, we note that, although only the $\mathrm{H} 1 s$ orbital is treated within DMFT, an interacting picture of the Ge orbitals (taking into account not only the Hubbard model interactions considered here but other, more general and perhaps nonlocal terms) is necessary to give the correct gapped band structure. In our DMFT calculations, this is accomplished through the eTB Hamiltonian, but here it is necessary to exclude the screening from the Ge $s p$ orbitals in order to get the most accurate assessment of the screened interactions on the $\mathrm{H}$ orbital. The LDA electronic structure (Fig. 1) in Sec. III A makes clear another reason for the necessity of treating the entire Ge $s p+\mathrm{H} s$ subspace within the cRPA: the $\mathrm{H} 1 s$ state is thoroughly entangled in the Ge $4 s$ and $4 p$ background. In fact, it appears to be split across two bands, frustrating attempts to isolate and manipulate it. Naturally, excluding the Ge $s p$ screening increases the resulting value of the $\mathrm{H} 1 s$ Hubbard $U$, which can be considered as an upper limit, and so the value of $U$ most appropriate for our $\mathrm{HGe}_{8}$ DMFT calculations (in which we only treat the dynamical correlations on the $\mathrm{H} 1 s$ orbital) is likely smaller than the $11.2 \mathrm{eV}$ we report in Table I. Due to the difference in correlated subspaces considered and the many varied parameters in the DMFT treatment, it is most appropriate to view this cRPA analysis as a separate method for understanding the $\mathrm{H}$ impurities and as a way to check
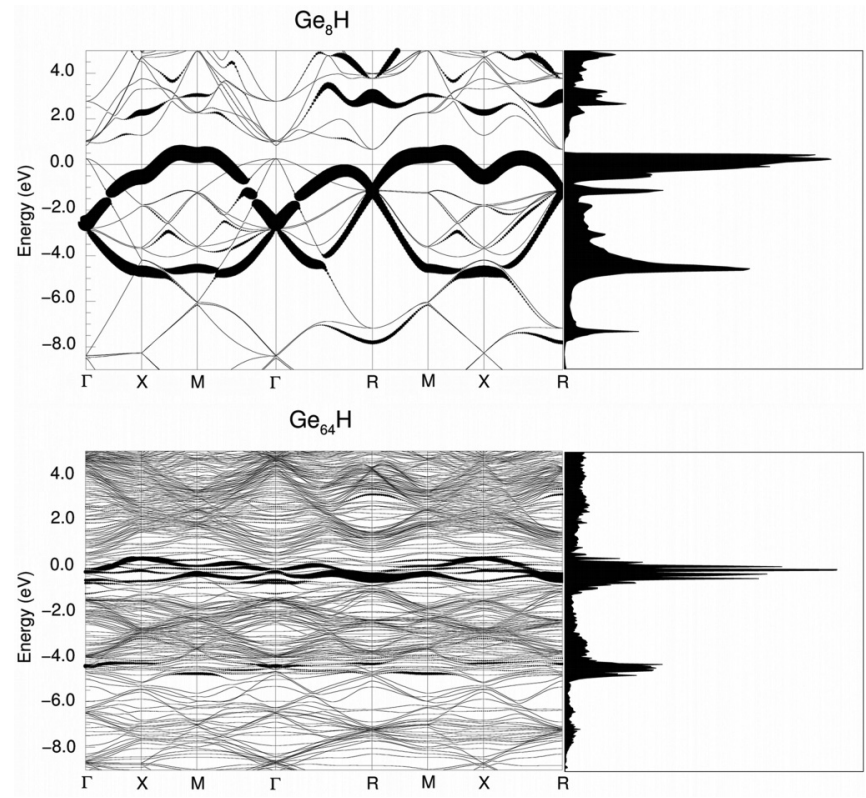

FIG. 1. Left panels: Fatband plots from LDA emphasizing the $\mathrm{H}$ $1 s$ character, indicated by the width of the band. Right panels: $\mathrm{H} 1 s$ projected density of states from the same calculation. For the $\mathrm{HGe}_{8}$ cell (top panels) seemingly two $1 s$ bands appear, with a total width of nearly $6 \mathrm{eV}$. The dispersion that is easy to follow reflects $\mathrm{H}-\mathrm{H}$ interaction within this small cell. In the 64-Ge cell (lower panels), the $1 s$ spectral density is expelled from the large-Ge DOS region ( -4 to $-1 \mathrm{eV})$, giving a bifurcation into two peaks separated by $4-5 \mathrm{eV}$.

the reasonableness of our DMFT approach. We note that the bare (unscreened) value $U_{b}=16.9 \mathrm{eV}$ is satisfyingly close to the analytic result for $U_{0}$ for an isolated $\mathrm{H}$ atom $(5 / 4 \mathrm{Ry}=$ $17.01 \mathrm{eV})$, reflecting the accuracy of the codes.

\section{Atomic solver}

We employ a hybridization-expansion continuous-time (CT-HYB) quantum Monte Carlo impurity solver, ${ }^{38}$ taking advantage of the segment picture ${ }^{39}$ to simplify the computations. Our solver is based upon that of the ALPS project; ${ }^{40}$ we also make use of the ALPS parallel Monte Carlo scheduler. ${ }^{41} \mathrm{Al}-$ though the CT-HYB solver has many advantages compared to the interaction-expansion method (CT-INT), the one-electron self-energy calculated from CT-HYB is highly sensitive to Monte Carlo noise. The Dyson equation gives the difference between Green's functions obtained from different Monte Carlo simulations, preventing the error from canceling. Indeed,

TABLE I. cRPA parameters screened Hubbard $U$ and Hund's rule $J \quad\left(U=F^{0}=\frac{1}{(2 l+1)^{2}} \sum_{m, n} U_{m n ; m n}\right.$ and $J=\frac{1}{2 l(2 l+1)} \sum_{m \neq n} U_{m n ; n m}$, where $l=0$ and 1 for $s$ and $p$ orbitals, respectively) calculated for $\mathrm{H} 1 s$ and $\mathrm{Ge} 4 s, 4 p$ orbitals in $\mathrm{HGe}_{8}$, when all $s p$ transitions were excluded. When all transitions are excluded we obtain the bare (unscreened) value $U_{b}$ and $J_{b}$.

\begin{tabular}{lrrrr}
\hline \hline Orbital & $U$ & $J$ & $U_{b}$ & $J_{b}$ \\
\hline $\mathrm{H}(1 s)$ & 11.2 & & 16.9 & \\
$\mathrm{Ge}(4 s)$ & 7.6 & & 11.9 & \\
$\mathrm{Ge}(4 p)$ & 5.7 & 0.3 & 9.2 & 0.4 \\
\hline \hline
\end{tabular}


the error in the self-energy is proportional to the absolute error in the Monte Carlo simulation, ${ }^{42}$ becoming much larger than the actual data even at relatively low frequencies. Moreover, one cannot accurately determine other quantities that are sensitive to the Green's function and self-energy at all frequencies (such as the occupation of the orbitals). Recently, two complementary solutions to this problem have arisen. Boehnke et al. $^{43}$ showed that, by measuring the Green's functions in an orthogonal Legendre basis (limited to a relatively small number of polynomials), one can filter out the Monte Carlo noise without losing any accuracy in the computation of the Green's functions and self-energies. Hafermann et al. $^{42}$ derived an expression for the self-energy involving a quotient of Green's functions rather than a difference. In this formulation, the error in the self-energy is proportional to the relative Monte Carlo error, leading to greatly reduced error into high frequencies. One can combine these methods for further reduction in the error, and we have implemented both.

\section{E. eTB + DMFT Hamiltonian}

Our Hamiltonian is that of the Anderson impurity model with a multiorbital "bath," which becomes 256 orbitals for our large cell. It can be represented by the following matrix:

$$
H^{\mathbf{k}}=\left(\begin{array}{cc}
H_{\mathrm{Ge}}^{\mathbf{k}} & V^{\mathbf{k}} \\
\left(V^{\mathbf{k}}\right)^{\dagger} & H_{\mathrm{imp}}
\end{array}\right),
$$

where $H_{\mathrm{Ge}}^{\mathbf{k}}$ is the supercell Hamiltonian for Ge obtained from the tight-binding model with no additional interaction parameters included, $V^{\mathbf{k}}$ is the H-Ge hybridization strength, and

$$
H_{\mathrm{imp}}=\left(\epsilon_{H}-\mu\right)\left(\hat{n}_{1 s, \uparrow}+\hat{n}_{1 s, \downarrow}\right)+U \hat{n}_{\uparrow} \hat{n}_{\downarrow}
$$

is the hydrogen Hamiltonian. There is only a density-density type interaction for a single nondegenerate correlated orbital, as required by the segment formulation of the CT-HYB method. Real frequency spectra are obtained using the maximum entropy (MaxEnt) method ${ }^{44}$ as implemented in the ALPS package. Static observables such as the average occupation $\langle n\rangle$, double occupation $\left\langle n_{\uparrow} n_{\downarrow}\right\rangle$, and square of the $z$ component of the spin magnetic moment $\left\langle m_{z}^{2}\right\rangle$ were measured during the Monte Carlo simulation $\left(m_{z} \equiv n_{\uparrow}-n_{\downarrow}\right)$.

\section{RESULTS AND DISCUSSION}

We present here results relating to a $\mathrm{H}$-like impurity in $\mathrm{Ge}$ as a function of the interaction strength $U$, the magnitude of the $\mathrm{H}-\mathrm{Ge}$ S-K hopping amplitude, and $\epsilon_{H}$, at both a 1:8 and 1:64 H-to-Ge ratio. We have considered temperatures ranging from $\beta=5 \mathrm{eV}^{-1}(T \approx 2300 \mathrm{~K})$ to $\beta=40 \mathrm{eV}^{-1}(T \approx 290 \mathrm{~K})$. One general observation is that the structure of the spectra that we obtain does not depend very significantly on temperature, so we will neglect temperature dependence in our discussion. In principle, the existence and character of gap states could show significant temperature dependence.

\section{A. LDA and $U=0$}

As presented in the LDA electronic structure of Fig. 1 obtained with the FPLO code ${ }^{45}$ the bands with predominantly $\mathrm{H} 1 s$ character can be readily identified. In both the $\mathrm{HGe}_{8}$ and $\mathrm{HGe}_{64}$ supercells, the $\mathrm{H} 1 s$ local density of states (DOS) bifurcates into two peaks, one in and just below the gap and the other around $-5 \mathrm{eV}$. Whereas these peaks are effectively separate in the larger cell, they form the boundaries of a broad but bifurcated $1 s$ bandwidth of bandwidth $W \approx 5-6 \mathrm{eV}$. The $\mathrm{H}$ spectral density is strongly expelled by the hybridization $V^{\mathbf{k}}$ from the large Ge DOS region between these peaks, leaving no clear way to identify an on-site $\mathrm{H} 1 s$ energy $\epsilon_{H}$.

The $\mathrm{HGe}_{8}$ cell result is anomalous in that $\mathrm{H}$ appears to introduce two new bands (the system is spin degenerate) in the system, whereas there is but a single $1 s$ orbital. The substantial dispersion of both bands indicates that $\mathrm{H}$ interstitials at this concentration are strongly coupled, representing an ordered alloy rather than an isolated impurity. The band structure is in fact metallic, with the upper $1 s$ band partially filled. The occurrence of strong $1 \mathrm{~s}$ character in two bands is clarified by the results of the $\mathrm{HGe}_{64}$ cell: some of the $1 s$ character $(\sim 25 \%)$ of the character lies $4-5 \mathrm{eV}$ below the gap, with the remaining spectral density lying just below the gap and slightly straddling it. The $\mathrm{H}$ spectral density within LDA is, as noted above, repelled from the region of large Ge $4 p$ DOS, with part going just below and the majority being pushed near the gap region. This bifurcation of spectral density may account for the fact that correlated band theory (the LDA + U method) was unable to produce a single magnetic hyperdeep state ${ }^{46}$ around $\sim-5 \mathrm{eV}$, as would be anticipated from the LDA $+\mathrm{U}$ method. The $\mathrm{HGe}_{64}$ results suggest this large cell is effectively in the isolated impurity limit.

We next survey the noninteracting spectrum (Fig. 2) within our eTB + DMFT picture (using the $\mathrm{S}-\mathrm{K}$ parameters displayed in Table II). We emphasize that this method is not equivalent to the LDA results just presented; most notably, in $\mathrm{HGe}_{64}$, it contains a gap whereas the LDA bands do not. The gap in fact is larger than the observed value for $\mathrm{Ge}$, but this allows us to assess more confidently the tendency toward formation of a gap state in the type of system we are studying: a $\mathrm{H}$-like interstitial impurity in a Ge-like semiconductor, rather than specifically $\mathrm{H}$ in $\mathrm{Ge}$. At reduced $\mathrm{H}-\mathrm{Ge}$ hybridization (dashed red lines in Fig. 2) the spectrum is dominated by a single Gaussian-like peak centered at $\epsilon_{H}$ with only small hybridization effects visible just below and just above the Fermi level, illustrating that the reduced hopping case indeed strongly reduces band structure signatures. At full hopping (solid black lines in Fig. 2), the spectrum is substantially spread into a large band with peaks and subpeaks arising from

TABLE II. Ge S-K empirical tight-binding parameters (in $\mathrm{eV}$ ) obtained from Newman and Dow, ${ }^{23}$ with $\mathrm{H}-\mathrm{Ge}$ eTB parameters taken from Pandey. ${ }^{24}$ The $\mathrm{H} s$ on-site parameter is varied in this study, as are the H-Ge hopping parameters; see the text.

\begin{tabular}{lcccccc}
\hline \hline & $E_{s}$ & $E_{p}$ & $V_{s s \sigma}$ & $V_{s p \sigma}$ & $V_{p p \sigma}$ & $V_{p p \pi}$ \\
\hline $\mathrm{Ge}$ & -5.8 & 1.61 & -1.695 & -2.03 & 2.65 & -0.67 \\
$\mathrm{H}-\mathrm{Ge}$ & $*$ & - & -3.30 & 2.16 & - & - \\
\hline \hline
\end{tabular}




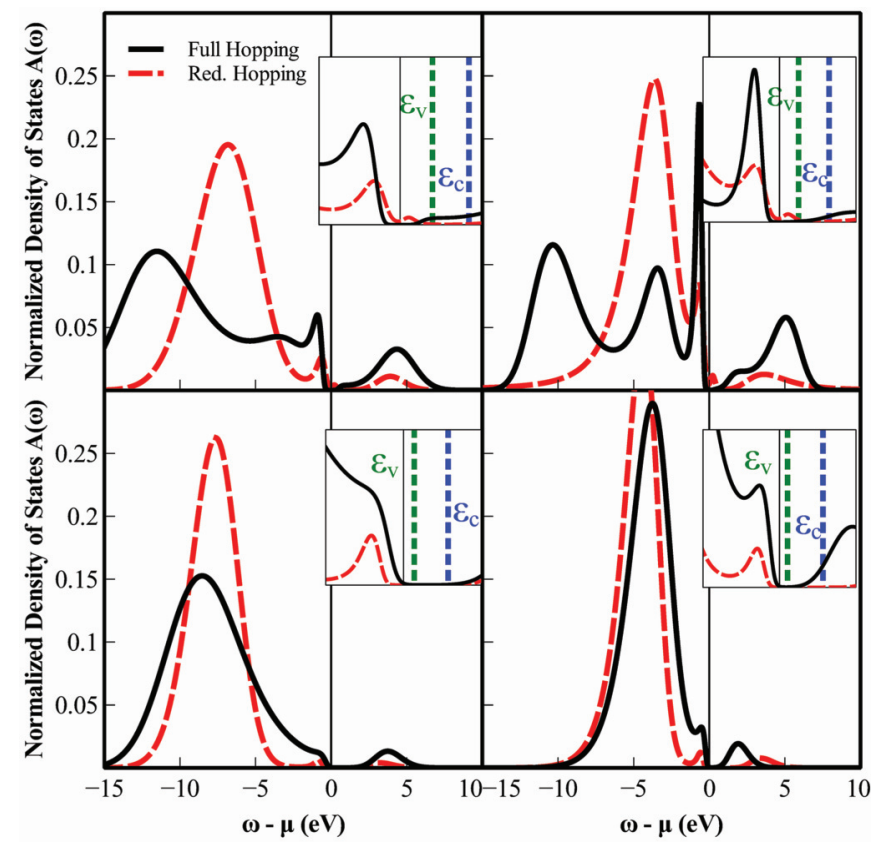

FIG. 2. (Color online) The $\mathrm{H} 1 s$ spectral density resulting from eTB + DMFT with $U=0$, i.e., before turning on the interaction. Results are shown for the two supercells $\left(\mathrm{HGe}_{8}\right.$, top panels; $\mathrm{HGe}_{64}$, bottom panels), and for $\epsilon_{H}=-8 \mathrm{eV}$ (left panels) and $-5 \mathrm{eV}$ (right panels). Reducing the $\mathrm{H}-\mathrm{Ge}$ hybridization from its full value (full line) leaves a spectrum (dashed line) that is dominated by a Gaussianlike peak centered on $\epsilon_{H}$. The insets provide an enlargement of the -2 to $+2 \mathrm{eV}$ regions, with the Ge band edges indicated by the dotted lines labeled $\epsilon_{v}$ and $\epsilon_{c}$.

hybridization between the $\mathrm{H} 1 s$ and Ge $4 s$ and $4 p$ states, with more of the weight appearing above the gap and well below $\epsilon_{H}$. As in the LDA results, the full-hybridization $\mathrm{HGe}_{8}$ calculations show that the $\mathrm{H} 1 s$ spectral density is expelled from the region with the largest Ge DOS. As anticipated, when $\epsilon_{H}$ is more shallow $(-5 \mathrm{eV})$ the spectral density shows more weight and structure near the gap. Note that without magnetic order or strong correlation effects (viz. in LDA) the Fermi level must fall within the bands, because our supercells contain an odd number of electrons which cannot be insulating with spin degeneracy. The insets in Fig. 2 show the position of the Ge valence and conduction band edges $\epsilon_{v}$ and $\epsilon_{c}$ in the full-hybridization calculations (we found that the Ge band edges did not depend strongly on the hybridization, so the half-hybridization values are suppressed for clarity).

The small upward shifts of the Ge bands can be understood by considering the occupancy of the $\mathrm{H}$ orbital. Tables III and IV provide the mean occupancy $\langle n\rangle$, double occupancy $\left\langle n_{\uparrow} n_{\downarrow}\right\rangle$, and the mean-square moment $\left\langle m_{z}^{2}\right\rangle$ (which is also the local susceptibility) for all cases studied. The $1 s$ occupation approaches two electrons in the absence of on-site Coulomb interactions, with the occupation increasing when the impurity level lies deeper and the hybridization is reduced. When $\langle n\rangle>1$, all Ge states cannot be occupied as just mentioned above, so slight hole doping will occur, leading to a weak acceptor picture. Van de Walle and Neugebauer obtained this type of result ${ }^{18}$ in their LDA studies of isolated $\mathrm{H}$ in Ge. In the smaller cell, the additional electron density is drawn from all Ge atoms. In the large cell, charge neutrality is accommodated by relatively small reorganization of electron density on the nearby Ge sites.

\section{B. $U=7 \mathrm{eV}$}

In the absence of guidance from past work, it seemed reasonable to choose interaction-strength $U$ values (reduced from the bare value) that highlight likely points of interest in the relationship between impurity energy level, hybridization strength, and on-site interactions. Choosing $U=7 \mathrm{eV}$ probes the behavior when $\epsilon_{H}+U<0$ as well as when $\epsilon_{H}+U>0$, given our two choices for the $1 s$ level. Note that in a mean-field treatment of the interaction, the effective (not bare) $\mathrm{H} 1 s$ level would be at $\epsilon_{H}+U\langle n\rangle / 2$ (since $\left.\left\langle n_{\uparrow}\right\rangle=\left\langle n_{\downarrow}\right\rangle=\langle n\rangle / 2\right)$.

Figure 3 shows the spectral functions at $U=7 \mathrm{eV}$, characterized by a large transfer of the spectral weight from $\epsilon_{H}$ at $U=0$ toward the gap region. It is possible that the gap survives in the $\epsilon_{H}=-8 \mathrm{eV}, \mathrm{HGe}_{64}$ spectra, but the imaginary-time and Matsubara Green's functions (not pictured) suggest that we should believe the small but finite DOS at the Fermi level observed in the MaxEnt data. This dominant effect of

TABLE III. Local quantities measured during the CT-HYB simulation for the $\mathrm{HGe}_{8}$ supercell. "Reduced" hopping signifies a tight-binding Hamiltonian in which the H-Ge S-K hopping parameters have been reduced to $50 \%$ of the value taken from Ref. 24 . The double-occupation and local moment show a strong dependence on the magnitude of the $\mathrm{H}-\mathrm{Ge}$ hopping and the position $\epsilon_{H}$ of the $1 \mathrm{~s}$ level. At half hopping, a large local moment arises for $U=7 \mathrm{eV}$ in the shallower state, with only a small increase in $\left\langle m_{z}^{2}\right\rangle$ as $U$ increases to $12 \mathrm{eV}$.

\begin{tabular}{|c|c|c|c|c|c|}
\hline \multirow[b]{2}{*}{$H G e_{8}$} & & \multicolumn{2}{|c|}{$\epsilon_{H}=-5 \mathrm{eV}$} & \multicolumn{2}{|c|}{$\epsilon_{H}=-8 \mathrm{eV}$} \\
\hline & & Full hopping & Reduced hopping & Full hopping & Reduced hopping \\
\hline \multirow[t]{3}{*}{$U=0 \mathrm{eV}$} & $\langle n\rangle$ & 1.66 & 1.89 & 1.79 & 1.94 \\
\hline & $\left\langle n_{\uparrow} n_{\downarrow}\right\rangle$ & 0.69 & 0.89 & 0.80 & 0.94 \\
\hline & $\left\langle m_{z}^{2}\right\rangle$ & 0.28 & 0.11 & 0.19 & 0.06 \\
\hline \multirow[t]{3}{*}{$U=7 \mathrm{eV}$} & $\langle n\rangle$ & 1.04 & 1.04 & 1.30 & 1.60 \\
\hline & $\left\langle n_{\uparrow} n_{\downarrow}\right\rangle$ & 0.21 & 0.12 & 0.38 & 0.60 \\
\hline & $\left\langle m_{z}^{2}\right\rangle$ & 0.62 & 0.80 & 0.54 & 0.40 \\
\hline \multirow[t]{3}{*}{$U=12 \mathrm{eV}$} & $\langle n\rangle$ & 0.90 & 0.99 & 1.06 & 1.06 \\
\hline & $\left\langle n_{\uparrow} n_{\downarrow}\right\rangle$ & 0.11 & 0.04 & 0.18 & 0.08 \\
\hline & $\left\langle m_{z}^{2}\right\rangle$ & 0.68 & 0.91 & 0.70 & 0.90 \\
\hline
\end{tabular}


TABLE IV. As in Table III, but for the $\mathrm{HGe}_{64}$ supercell. Compared to the smaller cell, the local moment state persists even at full $\mathrm{H}-\mathrm{Ge}$ hybridization and emerges at smaller values of $U$.

\begin{tabular}{|c|c|c|c|c|c|}
\hline \multirow[b]{2}{*}{$H G e_{64}$} & & \multicolumn{2}{|c|}{$\epsilon_{H}=-5 \mathrm{eV}$} & \multicolumn{2}{|c|}{$\epsilon_{H}=-8 \mathrm{eV}$} \\
\hline & & Full hopping & Reduced hopping & Full hopping & Reduced hopping \\
\hline \multirow[t]{3}{*}{$U=0 \mathrm{eV}$} & $\langle n\rangle$ & 1.94 & 1.97 & 1.94 & 1.98 \\
\hline & $\left\langle n_{\uparrow} n_{\downarrow}\right\rangle$ & 0.94 & 0.97 & 0.94 & 0.98 \\
\hline & $\left\langle m_{z}^{2}\right\rangle$ & 0.06 & 0.03 & 0.06 & 0.01 \\
\hline \multirow[t]{3}{*}{$U=7 \mathrm{eV}$} & $\langle n\rangle$ & 1.08 & 1.09 & 1.61 & 1.80 \\
\hline & $\left\langle n_{\uparrow} n_{\downarrow}\right\rangle$ & 0.14 & 0.10 & 0.62 & 0.81 \\
\hline & $\left\langle m_{z}^{2}\right\rangle$ & 0.80 & 0.89 & 0.37 & 0.18 \\
\hline \multirow[t]{3}{*}{$U=12 \mathrm{eV}$} & $\langle n\rangle$ & 0.99 & 1.00 & 1.08 & 1.02 \\
\hline & $\left\langle n_{\uparrow} n_{\downarrow}\right\rangle$ & 0.05 & 0.01 & 0.11 & 0.02 \\
\hline & $\left\langle m_{z}^{2}\right\rangle$ & 0.89 & 0.98 & 0.86 & 0.98 \\
\hline
\end{tabular}

$U$ contrasts the modest dependence on the H-Ge hopping strength, which is mostly observed in the degree of splitting in the lower "Hubbard subbands" and between the central peak and the upper and lower bands. This spectrum shift is accompanied by reduced $1 s$ occupation as expected (see Tables III and IV). At $\epsilon_{H}=-8 \mathrm{eV}$, the Ge valence band edge remains above $\mu$ due to the substantial $\mathrm{H} 1 s$ occupation, with the $\mathrm{H}$ gap state sitting just below $\epsilon_{v}$ in both supercells. In contrast, the $\epsilon_{H}=-5 \mathrm{eV}$ state is nearly singly occupied in both the small and large supercells, which allows $\mu$ to remain

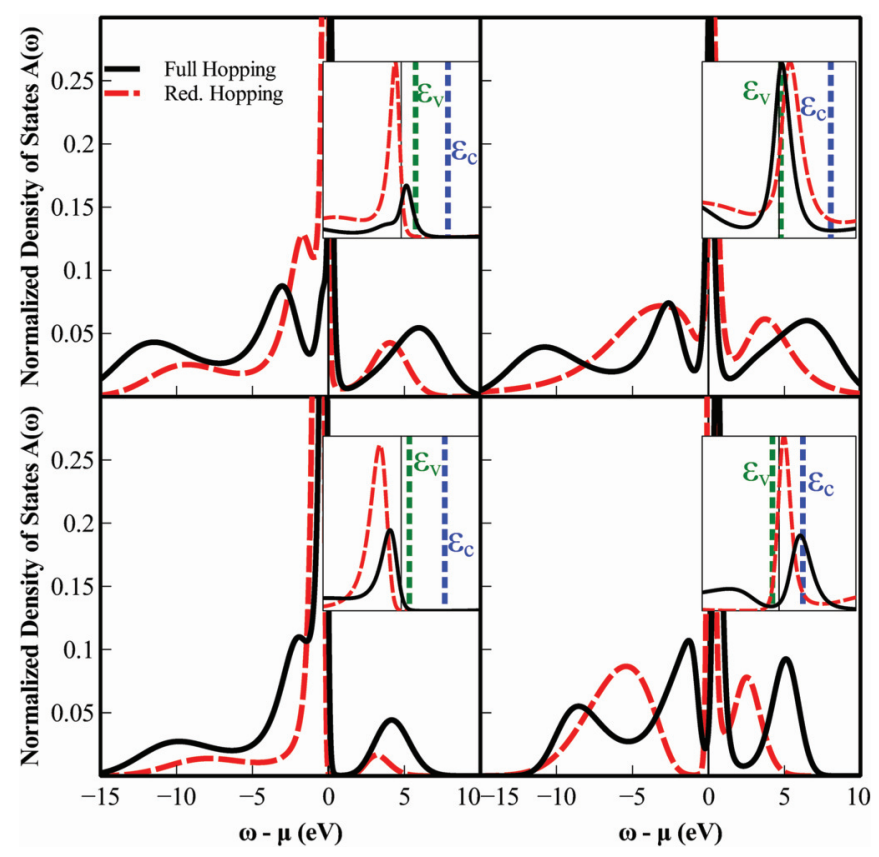

FIG. 3. (Color online) The introduction of an on-site Coulomb $U=7 \mathrm{eV}$ shifts most of the spectral weight to the vicinity of the Ge gap. For $\epsilon_{H}=-8 \mathrm{eV}$ (left), two spectral peaks are almost completely occupied, with the higher one overlapping a gap state (actual, or incipient) at $0 \mathrm{eV}$. The peak is more dramatic near the isolated impurity limit (lower left) and contains almost all of the $1 s$ density. The $\epsilon_{H}=$ $-5 \mathrm{eV}$ spectra (right) are characterized by a transfer of spectral weight to the Hubbard "bands" (now located on either side of the gap). (Inset) A view of the same MaxEnt spectra between -2 and $2 \mathrm{eV}$, with the Ge band edges indicated by the dotted lines labeled $\epsilon_{v}$ and $\epsilon_{c}$. at the top of the Ge valence band. Here, the $\mathrm{H}$ gap state sits near one of the band edges, falling squarely in the gap in $\mathrm{HGe}_{64}$.

At full hybridization strength, the $\mathrm{H}$ orbital occupation approaches half filling when $\epsilon_{H}=-5 \mathrm{eV}$, but the substantial double occupation leaves only a small local moment. For reduced hybridization the picture is different. In the $\epsilon_{H}=-8$ case, a small increase in $1 s$ occupation and a relatively large increase in $\left\langle n_{\uparrow} n_{\downarrow}\right\rangle$ yields a decrease in $\left\langle m_{z}^{2}\right\rangle$. With a shallower $\mathrm{H} 1 s$ level, however, the orbital remains close to half filling and the $1 s$ local moment grows much larger, tending to form a nearly fully-spin-polarized paramagnetic state. In the $\mathrm{HGe}_{64}$ cell, where $\mathrm{H}-\mathrm{H}$ interaction through the Ge states is negligible, a large local moment appears at $\epsilon_{H}=-5$ at both full and reduced hybridization.

Examining the placement of the gap state, we find that its position appears to be governed by several factors. It always sits at or near either the top of the Ge valence band or the bottom of the Ge conduction band, depending on which is closer to the Fermi level. Typically, the gap state sits at the top of the Ge valence band, but in some cases, charge neutrality dictates that $\mu$ moves into the Ge conduction states. Starting from the Ge band edge, the gap state's position is further determined by hybridization effects. At full hybridization, the gap state is usually deflected "upward" and away from the split lower Hubbard-like sub-bands. However, there is a similar repulsion of spectral weight due to the upper bands, and so the position is finally determined by the interplay of these factors.

$$
\text { C. } U=12 \mathrm{eV}
$$

Increasing the interaction strength to $U=12 \mathrm{eV}$, which is near the cRPA value, prompts some further spreading of the spectral weight and additional sharpening (but also shrinking) of the spectral peak in the gap region as the upper Hubbardlike band is pushed well clear. The five-peak structure that emerges in $\mathrm{HGe}_{8}$ can be understood by the splitting of the upper and lower Hubbard bands due to $\mathrm{H}-\mathrm{Ge}$ hybridization (leading to much reduced or absent splitting in the low-hopping case). The $1 s$ orbital tends toward half filling for all parameter values, reflecting the strong coupling limit. Qualitatively the $1 \mathrm{~s}$ spectrum in $\mathrm{HGe}_{8}$ is not affected greatly by the near doubling of the interaction strength. However, in Sec. III D, we find that $\left\langle m_{z}^{2}\right\rangle$ reaches its maximum value at or near $U=12 \mathrm{eV}$ at all values of $\epsilon_{H}$ and the $\mathrm{H}-\mathrm{Ge}$ hybridization that we consider. 


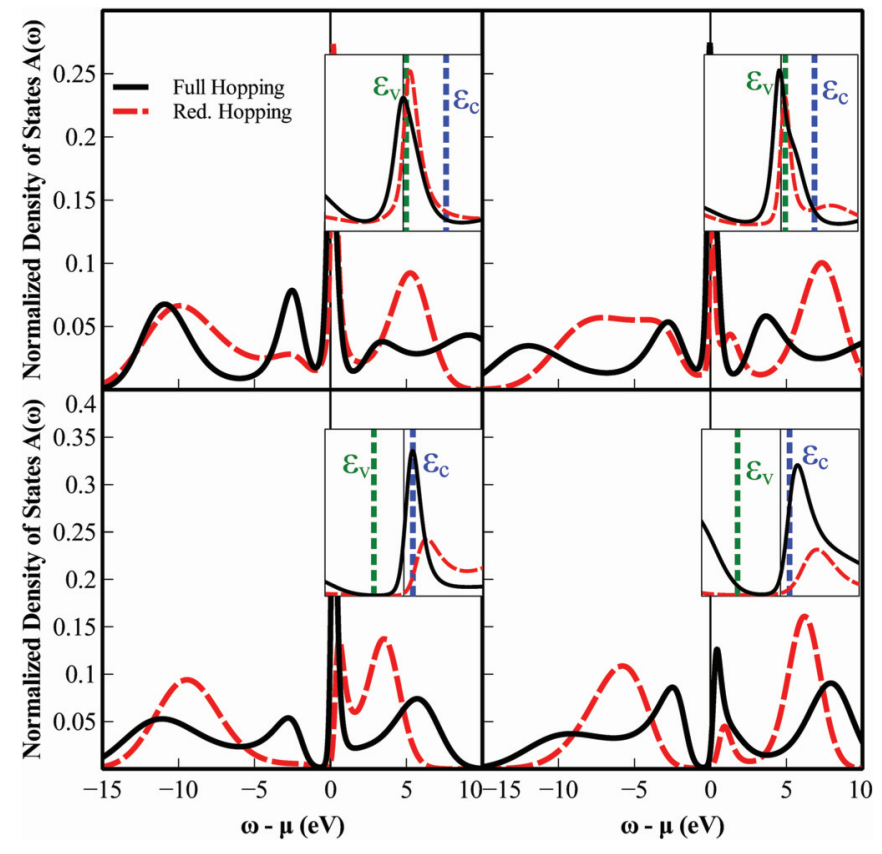

FIG. 4. (Color online) For strong interactions $(U=12 \mathrm{eV})$, the low-energy quasiparticlelike peak diminishes with respect to the states centered at $\epsilon_{H}$ and in the conduction band. This effect is most dramatic in the 64-Ge cell, where the spectral weight in the dominant peak at $U=7$ is almost completely transferred to higher energy, opening or nearly opening a clear gap. In all cases, the $1 s$ orbital approaches half filling and develops a local moment. (Insets) A view of the same MaxEnt spectra between -2 and $2 \mathrm{eV}$, with the Ge band edges indicated by the dotted lines labeled $\epsilon_{v}$ and $\epsilon_{c}$.

The $\mathrm{H} 1 s$ spectrum of $\mathrm{HGe}_{64}$ begins to differ more strongly from that of the smaller cell when the interaction becomes strong. A gap that roughly corresponds to the Ge gap but does not necessarily fall across $\mu$ is restored for both values of the $\mathrm{H}$ energy level (Fig. 4). Further, the sharp peak now just above the gap begins to dissipate, only just surviving in the $\epsilon_{H}=-8 \mathrm{eV}$ spectra at full hopping, while nearly disappearing for reduced hopping in the $\epsilon_{H}=-5$ spectra. This behavior suggests that a Mott-Hubbard insulating character of the $1 s$ spectrum should arise as the impurity limit is approached or as the interaction continues to increase.

\section{Transition into the local moment state}

Transitions to a magnetic local moment state $\left\langle m_{z}^{2}\right\rangle \sim \mathcal{O}(1)$ occur at different interaction strengths for different values of $\epsilon_{H}$ and $\mathrm{H}-\mathrm{Ge}$ hopping. To shed further light on this transition, we performed a more detailed set of calculations. We varied $U$ between 4 and $12 \mathrm{eV}, \epsilon_{H}$ between -5 and $-8 \mathrm{eV}$, and the $\mathrm{H}-\mathrm{Ge}$ hopping between $50 \%$ and $100 \%$ of the eTB value, with all calculations performed in the $\mathrm{HGe}_{8}$ cell. The results are summarized in Fig. 5.

At low $U$, the the local moment at $\frac{1}{2}$ hopping (dotted curve in Fig. 5) can be much smaller than when hybridization is larger. Table III shows that this is due to the large occupation of the $\mathrm{H}$ orbital, particularly when $\epsilon_{H}$ is below $-5 \mathrm{eV}$. As $U$ increases, the dashed and dotted curves $\left(\frac{3}{4}\right.$ and $\frac{1}{2}$ hopping, respectively) show much larger moments, with $\left\langle m_{z}^{2}\right\rangle$ in excess

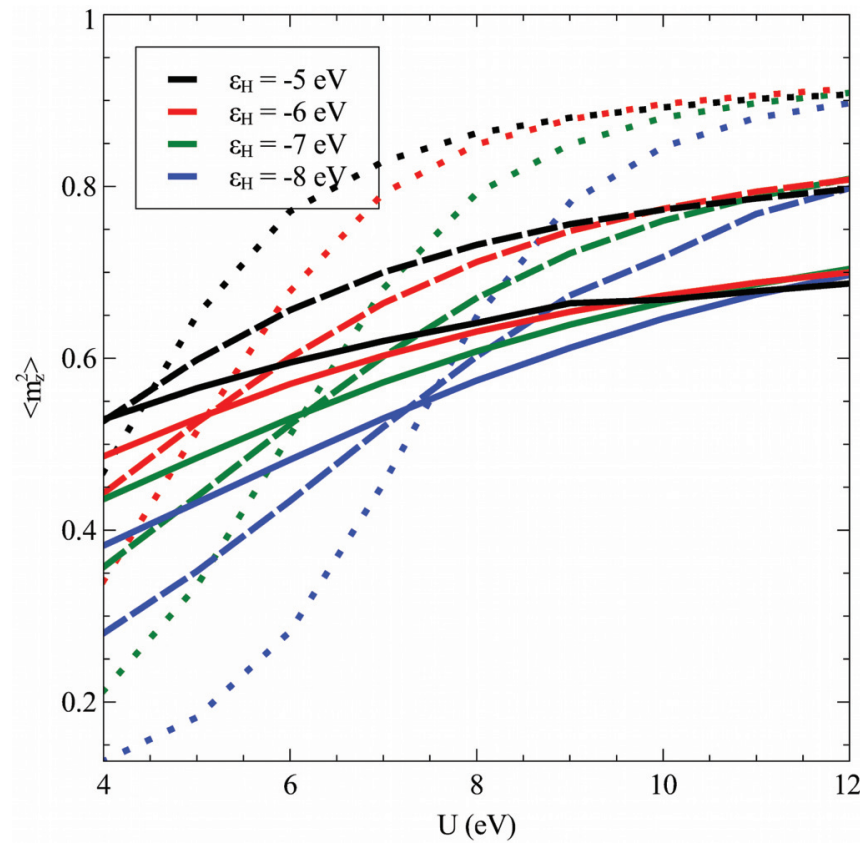

FIG. 5. (Color online) Monotonic increase of the $1 s$ moment on interaction strength $U$ for a range of $1 s$ level positions $\epsilon_{H}$, at full $\mathrm{H}-\mathrm{Ge}$ hopping amplitude (solid), $\frac{3}{4}$ hopping (dashed), and $\frac{1}{2}$ hopping (dotted). Reduction in H-Ge hybridization results, as expected, in greater spin polarization in the impurity orbital, but the effect becomes pronounced for reduction below $75 \%$ hopping amplitude where the dependence on $\epsilon_{H}$ also becomes strong.

of 0.9 in the $\mathrm{H}$ orbital at $\frac{1}{2}$ hopping. This behavior can be understood both as a direct result of the hybridization and the increase in interaction strength. The behavior of the curves at $U=12 \mathrm{eV}$ shows that the largest $U$ we considered in the main body of our DMFT study is sufficient to saturate the local moment: a fortuitous coincidence. Within each set of curves, it can be observed that until relatively large $U$, the local moment increases with decreasing $\mathrm{H}$ level depth. Both this trend and its trend toward reversal at very large $U$ arise from the orbital's proximity to half filling. Clearly an orbital at half filling is able to support a larger moment than one well away from half filling. Below $U \approx 8 \mathrm{eV}$, only the shallower states are able to push their upper "Hubbard bands" above the gap. At large $U$, on the other hand, the orbital approaches half filling regardless of $\epsilon_{H}$, although for the shallowest $1 s$ level, filling falls below unity accompanied by a smaller moment. This tendency to form moments at low effective hybridization and large $U$ is consistent with the infinite- $U, \mathrm{HGe}_{54}$ mean-field treatment of PCK, and with lore accumulated in the interim. In addition, this behavior bears some resemblance to that predicted by $\mathrm{Li}$ et $a l .{ }^{47}$ for impurities on graphene, where there is a vanishing gap and the environment is two rather than three dimensional.

\section{SUMMARY}

We have employed dynamical mean-field theory using the CT-HYB solver to study the electronic structure of one of the simplest, but still important conceptually and possibly technologically, impurity systems: an interstitial H-like impurity in the antibonding interstitial site in a diamond-structure covalent 
semiconductor representative of Ge. The nondegenerate $1 s$ orbital precludes any necessity of considering Hund's rule magnetic coupling or multiplet effects, so DMFT is the exact solution to the impurity problem within the limit of a large supercell. Because Ge within LDA has no gap, the noninteracting system was represented by an empirical tight-binding model with parameters based on previous $\mathrm{H}-\mathrm{Ge}$ studies.

The $\mathrm{H} 1 s$ spectrum shows a rich but systematic behavior as the on-site interaction parameter $U$, the H-Ge hopping amplitude $V^{\mathbf{k}}$, and the $\mathrm{H}$ on-site energy $\epsilon_{H}$ are varied, with some parameter ranges possibly giving some insight into Si or diamond hosts as well as Ge. The dependence on temperature was studied but found to be minor, and therefore has not been presented. Our results demonstrate that electron-electron correlations can play a role in determining the properties of the isolated $\mathrm{H}$ impurity in such a system. If $\epsilon_{H}+U>0$, which naively puts the upper "Hubbard band" above the gap, the on-site Coulomb repulsion is sufficient to prevent the $\mathrm{H}$ orbital from acquiring electrons from the surrounding Ge, as is the case within LDA.

The behavior of this system is enriched by the bifurcation, at the LDA level, of the $1 s$ spectrum, with much of the weight in and just below the gap and the rest around 4-5 eV binding energy. Within the extended tight-binding model we use and with $U=0$, which is not exactly the same as within LDA but is closely related, the $1 s$ occupation is 1.7-1.8, reflecting the acceptor character (hole doping) of Ge before the interaction is turned on. The distributed spectrum primarily through the valence region may be interpreted as the hyperdeep-donor character that was envisioned by PCK. Increasing $U$ and/or reducing the hybridization moves the $1 s$ occupation toward unity-Anderson impurity character-with the rate of approach affected by the choice of the bare $1 s$ site energy $\epsilon_{H}$.

To contribute to a more specific study of $\mathrm{H}$ in $\mathrm{Ge}$, we have computed the interaction parameters $U$ and $J$ in $\mathrm{HGe}_{8}$ for the
Ge $s p$ and $\mathrm{H} s$ orbitals within the constrained RPA formalism. The $\mathrm{H} 1 s$ Hubbard $U$ is, as expected, much larger than those associated with the Ge $4 s$ or $4 p$ states, and is coincidentally similar to the largest interaction parameter we considered in our DMFT approach. Further studies are necessary to pin down the behavior of a real $\mathrm{H}$ atom in $\mathrm{Ge}$, which will involve optimization of the energy versus $\mathrm{H}$ position together with relaxation of the $\mathrm{Ge}$ positions. In $\mathrm{Si}$, for example, $\mathrm{H}$ more commonly assumes a bond-center position, although the tetrahedral interstitial is not far above in energy. ${ }^{10}$ The methods needed for those calculations require accurate total energy capability with charge self-consistency, and must include the weak correlation within the Ge $s p$ bands that gets the band gap correct as well as the potentially moderately strong correlation associated with the $\mathrm{H}$ impurity. As such, this $\mathrm{H}-\mathrm{in}-\mathrm{Ge}$ problem poses a strong challenge for the future.

\section{ACKNOWLEDGMENTS}

H.S. wishes to acknowledge helpful conversations with L. Boehnke and H. Hafermann regarding their improved measurement techniques. W.E.P. acknowledges J. C. Smith for discussions of calculations of $\mathrm{H}$ interstitials in (insulating) diamond and xenon. Calculations were performed using computational resources from the University of Alabama MINT High Performance Cluster and the NICS Kraken Cray XT5 under XSEDE Project TG-PHY120018. W.E.P. acknowledges support from Department of Energy Stewardship Science Academic Alliances program under Grant No. DE-FG0303NA00071 and from the Simons Foundation, and E.R.Y. acknowledges support for algorithm development and implementation to the National Science Foundation program Physics at the Information Frontier through Grant No. PHY-1005503. E.Ş., C.F., and S.B. acknowledge the support of DFG through the Research Unit FOR-1346.
${ }^{1}$ F. D. M. Haldane and P. W. Anderson, Phys. Rev. B 13, 2553 (1976).

${ }^{2}$ W. E. Pickett, M. L. Cohen, and C. Kittel, Phys. Rev. B 20, 5050 (1979).

${ }^{3}$ E. E. Haller, Phys. Rev. Lett. 40, 584 (1978).

${ }^{4}$ M. Budde, B. Bech Nielsen, R. Jones, J. Goss, and S. Öberg, Phys. Rev. B 54, 5485 (1996).

${ }^{5}$ J. P. Goss, J. Phys.: Condens. Matter 15, R551 (2003).

${ }^{6}$ B. J. Coomer, P. Leary, M. Budde, B. Bech Nielsen, R. Jones, S. Öberg, and P. R. Briddon, Mater. Sci. Eng. B 58, 36 (1999).

${ }^{7}$ J. Weber, M. Hiller, and E. V. Lavrov, Mater. Sci. Semicond. Process. 9, 564 (2006).

${ }^{8}$ M. Budde, B. Bech Nielsen, C. Parks Cheney, N. H. Tolk, and L. C. Feldman, Phys. Rev. Lett. 85, 2965 (2000).

${ }^{9}$ C. P. Cheney, M. Budde, G. Lüpke, L. C. Feldman, and N. H. Tolk, Phys. Rev. B 65, 035214 (2002).

${ }^{10}$ E. A. Davis, J. Non-Cryst. Solids 198-200, 1 (1996).

${ }^{11}$ C. C. Yu and M. Guerrero, Phys. Rev. B 54, 8556 (1996).

${ }^{12}$ M. R. Galpin and D. E. Logan, Phys. Rev. B 77, 195108 (2008).

${ }^{13}$ M. R. Galpin and D. E. Logan, Eur. Phys. J. B 62, 129 (2008).

${ }^{14}$ W. Kohn and C. Majumdar, Phys. Rev. 138, A1617 (1965).

${ }^{15}$ J. Oliva and L. M. Falicov, Phys. Rev. B 28, 7366 (1983).
${ }^{16}$ J. Oliva, Phys. Rev. B 29, 6846 (1984).

${ }^{17}$ S. K. Estreicher and Dj. M. Maric, Phys. Rev. Lett. 70, 3963 (1993).

${ }^{18}$ C. G. van de Walle and J. Neugebauer, Nature (London) 423, 626 (2003).

${ }^{19}$ L. M. Almeida, J. Coutinho, V. J. B. Torres, R. Jones, and P. R. Briddon, Mater. Sci. Semicond. Proc. 9, 503 (2006).

${ }^{20}$ E. Müller-Hartmann, Z. Phys. B 74, 507 (1989).

${ }^{21}$ W. Metzner and D. Vollhardt, Phys. Rev. Lett. 62, 324 (1989).

${ }^{22}$ A. Georges and G. Kotliar, Phys. Rev. B 45, 6479 (1992).

${ }^{23}$ K. E. Newman and J. D. Dow, Phys. Rev. B 30, 1929 (1984).

${ }^{24}$ K. C. Pandey, Phys. Rev. B 14, 1557 (1976).

${ }^{25}$ F. Aryasetiawan, M. Imada, A. Georges, G. Kotliar, S. Biermann, and A. I. Lichtenstein, Phys. Rev. B 70, 195104 (2004); F. Aryasetiawan, K. Karlsson, O. Jepsen, and U. Schönberger, ibid. 74, 125106 (2006); T. Miyake, F. Aryasetiawan, and M. Imada, ibid. 80, 155134 (2009).

${ }^{26}$ B-C. Shih, Y. Zhang, W. Zhang, and P. Zhang, Phys. Rev. B 85, 045132 (2012).

${ }^{27}$ E. Şaşığlu, C. Friedrich, and S. Blügel, Phys. Rev. Lett. 109, 146401 (2012). 
${ }^{28}$ H. Sims, W. H. Butler, M. Richter, K. Koepernik, E. Şaşığlu, C. Friedrich, and S. Blügel, Phys. Rev. B 86, 174422 (2012).

${ }^{29}$ E. Şaşıŏlu, C. Friedrich, and S. Blügel, Phys. Rev. B 83, 121101(R) (2011).

${ }^{30}$ N. Marzari and D. Vanderbilt, Phys. Rev. B 56, 12847 (1997).

${ }^{31} \mathrm{http}$ ://www.flapw.de

${ }^{32}$ J. P. Perdew, K. Burke, and M. Ernzerhof, Phys. Rev. Lett. 77, 3865 (1996).

${ }^{33}$ F. Freimuth, Y. Mokrousov, D. Wortmann, S. Heinze, and S. Blügel, Phys. Rev. B 78, 035120 (2008).

${ }^{34}$ A. A. Mostofi, J. R. Yates, Y.-S. Lee, I. Souza, D. Vanderbilt, and N. Marzari, Comput. Phys. Commun. 178, 685 (2008).

${ }^{35}$ C. Friedrich, S. Blügel, and A. Schindlmayr, Phys. Rev. B 81, 125102 (2010).

${ }^{36}$ E. Şaşığlu, A. Schindlmayr, C. Friedrich, F. Freimuth, and S. Blügel, Phys. Rev. B 81, 054434 (2010).

${ }^{37}$ C. Friedrich, A. Schindlmayr, and S. Blügel, Comput. Phys. Commun. 180, 347 (2009).
${ }^{38}$ P. Werner and A. J. Millis, Phys. Rev. B 74, 155107 (2006).

${ }^{39}$ P. Werner, A. Comanac, L. de'Medici, M. Troyer, and A. J. Millis, Phys. Rev. Lett. 97, 076405 (2006).

${ }^{40}$ E. Gull, P. Werner, S. Fuchs, B. Surer, T. Pruschke, and M. Troyer, Comput. Phys. Commun. 182, 1078 (2011).

${ }^{41}$ M. Troyer, B. Ammon, and E. Heeb, Lect. Notes Comput. Sci. 1505, 191 (1998).

${ }^{42}$ H. Hafermann, K. R. Patton, and P. Werner, Phys. Rev. B 85, 205106 (2012).

${ }^{43}$ L. Boehnke, H. Hafermann, M. Ferrero, F. Lechermann, and O. Parcollet, Phys. Rev. B 84, 075145 (2011).

${ }^{44}$ R. N. Silver, D. S. Sivia, and J. E. Gubernatis, Phys. Rev. B 41, 2380 (1990); M. Jarrell and O. Biham, Phys. Rev. Lett. 63, 2504 (1989).

${ }^{45}$ K. Koepernik and H. Eschrig, Phys. Rev. B 59, 1743 (1999); http://www.fplo.de.

${ }^{46}$ J. C. Smith and W. E. Pickett (unpublished).

${ }^{47}$ C. Li, J.-X. Zhu, and C. S. Ting, arXiv:1106.5827v1. 